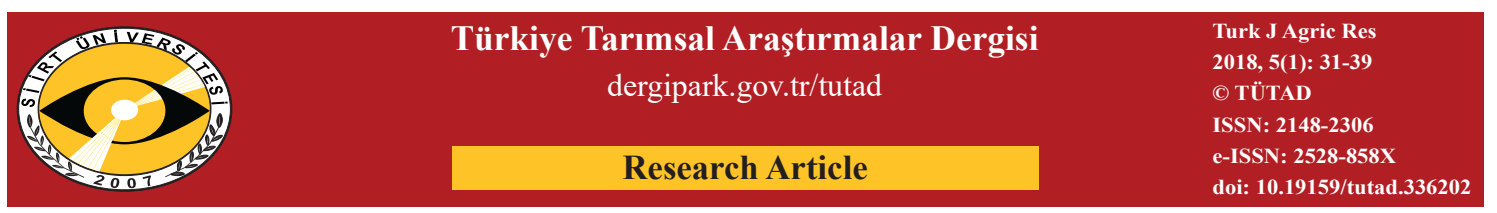

\title{
In Silico Analysis of Mobilome Response to Salt Stress in Phaseolus vulgaris L.
}

\author{
Behcet İNAL* \\ Siirt University, Faculty of Agriculture, Department of Agricultural Biotechnology, Siirt, TURKEY
}

\begin{tabular}{l}
\hline Received: $26.08 .2017 \quad$ Accepted: 24.11 .2017 \\
\hline ORCID ID \\
(Dorcid.org/0000-0003-2215-2710 \\
${ }^{*}$ Corresponding Author: behcetinal@siirt.edu.tr
\end{tabular}

\begin{abstract}
Common bean is an important legume that grown and consumed as animal feed and for human nutrition. It is also an important source of protein in developing countries. Transposable elements (TEs) constitute a large part of the genome in various eukaryotic species. TE was described as garbage DNA by researchers for a long time. Recently, it has been found that TEs can move near stress response genes and they have known effects on plant resistance to diverse stresses. With the acquisition of common bean genome sequence, one of the next step is to annotate the genome and define the functional DNA elements. TEs are the most abundant genetic elements of plant genomes and have an important impact on genome stress evolution and genetic variation. So, it is important to determine TEs in the common bean genome. In the current study, genome-wide transposon annotation and definition were achieved in root and leaf tissues of common bean under salt stress. Homology and sequence structure-based methods were used. Tont2-I-Copia and Copia-39 Copia retrotransposons were found to be more in salt-treated roots and leaves respectively. As a result of the analysis, we found TEs number ranging from 46 to 50 belonging to about twenty different plants. Gene ontology analysis of transposon sequences brought the light on diverse important pathways related to abiotic stress conditions.
\end{abstract}

Keywords: Abiotic stress, common bean, gene ontology, genome-wide, transposon

\section{Introduction}

Common bean (Phaseolus vulgaris L., $2 \mathrm{n}=2 \mathrm{x}=22$ ) is an important legume grown throughout the world and can also be consumed as an animal feed because of rich protein ingredient. In some developing countries, common bean is an important source of economic income (Blair et al., 2011). Common bean supply micronutrients, proteins, fibers and other considerable components which are unavailable or in low levels in other crops. It also is a member of legume family which are the most worthy for their capability to fix atmospheric nitrogen through symbiosis with soil-borne rhizobia (Lin et al., 2010; Bitocchi et al., 2012; McClean et al., 2010).

Transposable elements (TEs), first identified as "controlling elements" by Barbara McClintock (McClintock, 1956), are now believed to compose a large part of the angiosperm genome (Biémont,
2010; Wicker et al., 2007). TE causes mutant alleles by altering the reading frame or splicing profile, frequently adversely influencing gene function. However, TEs also have a pivotal impact on the regulation of gene expression, potentially can affect the molecular changing in responses to environmental stress (McClintock, 1984). McClintock initially defined the TEs as "controlling elements" because of their capability in effecting expression of nearby genes (McClintock, 1963). Many specific TEs that regulate the expression of nearby genes have been known (Desouza et al., 2013; Feschotte, 2008; Cowley, 2013). TE insertions near genes may affect gene regulation through several potential approaches, including join in cis-regulatory regions, supplying novel cis-regulatory sequences that can play role as enhancers/repressors by simplifying transcription factor binding (Ito et al., 2011), or may have an impact on chromatin region of gene 
promoter (Eichten et al., 2012; Hollister and Gaut, 2009). Many TEs serve as stress-responsive transcriptions (Hollister and Gaut, 2009; Ito et al., 2013). For instance, expression of the tobacco Tnt 1 genetic element can be triggered by biotic and abiotic stress (Beguiristain et al., 2001). DNA transposon mPing of Rice (Oryza sativa) can be induced under cold and salt stress conditions (Yasuda et al., 2013). The ONSEN retrotransposon of Arabidopsis activated transcriptionally during the heat stress (Pecinka et al., 2010; Cavrak et al., 2014). These studies indicates that TEs can exhibit novel regulatory mechanisms and have an impact on the response to environmental stresses (Ito et al., 2013). Grandbastien et al. (1997) reported that the expression of Tto 1 and Tnt1 retrotransposons rose in tobacco plant subjected to salt stress. Sigmaz et al. (2015) also indicated that salt stress caused LTR retrotransposon polymorphisms in Triticum aestivum $\mathrm{L}$.

In the current study, a subset of TE families responsive to salt stress in common bean, based on genome-wide transposon homology and annotation, was defined. According to our data, TEs genes can exhibit salt stress-responsive regulation of gene expression. It was also found that stress-responsive TEs appears to exhibit many important gene ontology and pathways that strongly associated with response to salt stress in common bean.

\section{Materials and Methods}

\subsection{RNA-Seq data use in study}

Illumina RNA-Seq data was obtained from Sequence Read Archive (SRA) in order to define the TEs. The accessions SRR957667 (LC: control leaf), SRR958472 (RT: root treated with salt-), SRR958469 (RC: root control), and SRR957668 (LT: leaf treated with salt) were used (Hiz et al., 2014). All readings were obtained in raw sequence data as ".sra" format and converted to "fastq" format for Illumina by the NCBI (National Center for Biotechnology Information) SRA Toolkit. After cutting the low-quality readings (Phred quality (Q) score $<20$ ) and trimming adapters with FASTX toolkit, all clean readings were exposed to FastQC analysis to control reading qualities in terms of perbase sequence qualities. The raw data count was transformed and normalized by using the CLC Genomics Workbench 10.1.1. All readings were assembled by using the Trinity software (Haas et al., 2013).

\subsection{Defining transposon elements responsive to salt stress}

To identify the transposons responsive to salt stress, we used Basic Local Alignment Search Tool
(BlastN 2.6. 0) to search against all sequences of transposons in Repbase database by using 10-5 Evalue as a cutoff point. Repbase is known as a worldwide reference standard for annotating the existence of repetitive DNA in genomic data (Jurka et al., 2005). Venn diagram was obtained by using the Venny tool (Anonymous, 2017).

\subsection{GO annotation of transposon elements}

The functional annotation of salt stress response TEs sequences and the analysis of annotation data were subjected to Blast2GO (Conesa et al., 2005). First, all identified TEs DNA sequences of LC, LT, $\mathrm{RC}, \mathrm{RT}$ tissues were exposed to Blast2GO software. Then, functional annotation was performed in three steps: (1) BlastP to achieve the homologous sequences, (2) MAPPING to get the Gene Ontology (GO) terms related with the Blast results, and (3) ANNOTATION of GO terms respectively.

\section{Result and Discussion}

\subsection{Sequence quality use in study}

RNA-Seq data that used in the study was generated by Illumina next-generation sequencing platform (Hiz et al., 2014). Sequence data was composed of four libraries of salt treated leaf and root tissues. After read assembling process with Trinity algorithm (Haas et al., 2013), sequence lengths were found to be between 500-3099 base pair (bp) (Figure 1). These parameters of RNA-Seq were assessed quite significant and confident for performing further in silico analysis such as TEs meta-analysis. Additionally, many previous NGS studies obtained almost similar results (Li et al., 2016; Li et al., 2015). LC, LT, RC and RT libraries had 72.852, 68.960, 79.009 and 75.893 sequences, respectively and a total of 201.264 .347 nucleotides were used to infer TEs in common bean genome (Table 1). These sequence numbers were found to be enough for in-silico analysis of salt stress response TEs in common bean.

\subsection{Detection of TEs response to salt stress}

To identify the TEs responsive to salt stress in common bean, BlastN search was performed against all sequences of transposons in Repbase database. TE was found at various number levels ranging between 46 to 50 belonging to twenty different plant species: These species are Arabidopsis thaliana, Arachis ipaensis, Brachypodium distachyon, Branchiostoma floridae, Callorhinchus milii, Cicer arietinum, Esox lucius, Fragaria vesca, Glycine max, Gossypium raimondii, Jatropha curcas, Medicago truncatula, Melopsittacus undulatus, Oryza sativa, Phaseolus vulgaris, Populus trichocarpa, Pyrus $x$ 

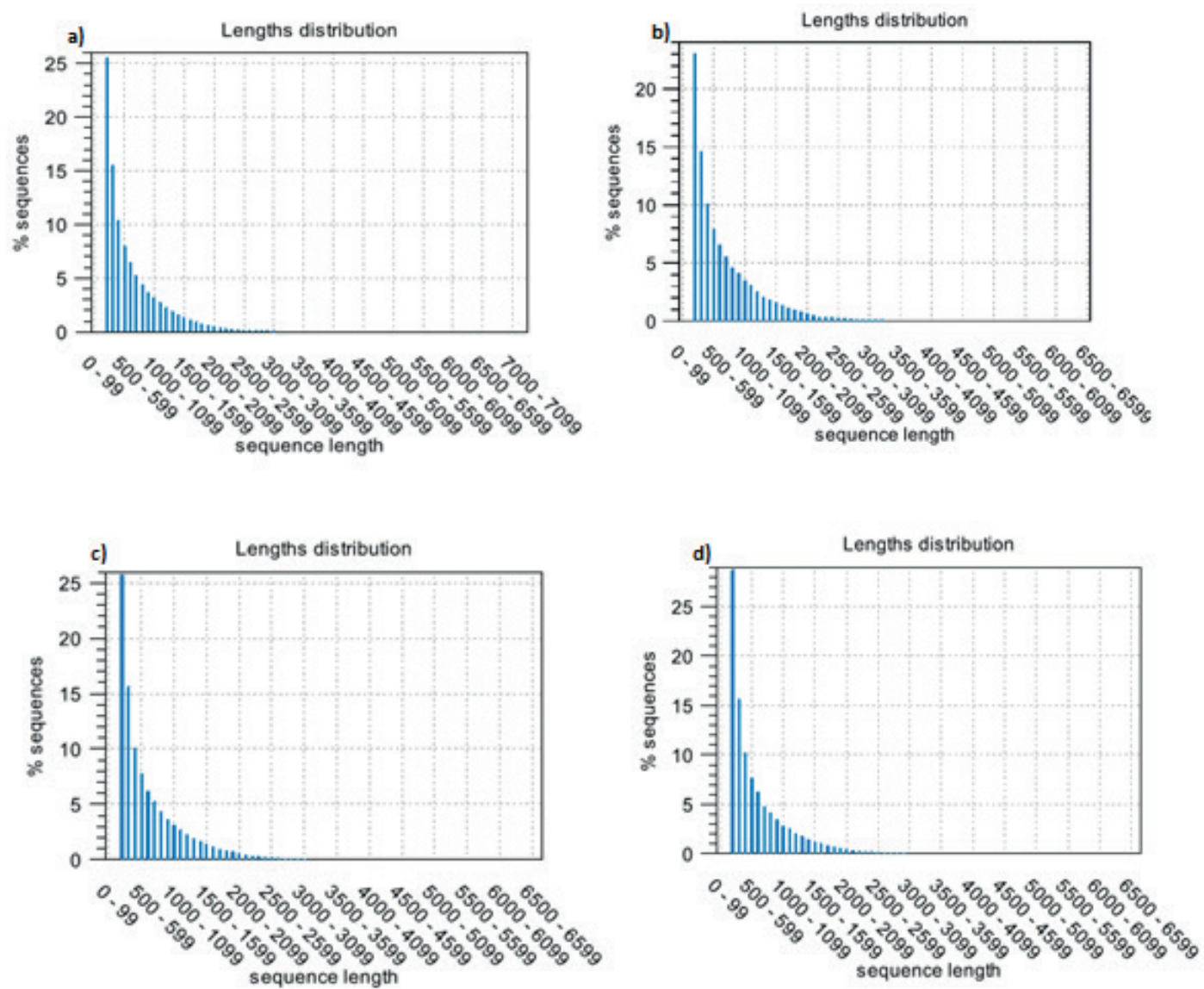

Figure 1. Sequence and read length distribution of four RNA-Seq libraries (a: LC, b: LT, c: RC, d: RT)

Table 1. General information about RNA-Seq libraries used in study

\begin{tabular}{ll}
\hline RNA-Seq library name & Number of sequences \\
\hline Leaf control & 72.852 \\
Leaf salt treated & 68.960 \\
Root control & 79.009 \\
Root salt treated & 75.893 \\
Total sequences in data sets & 296.714 \\
Total nucleotides in data & 201.264 .347 nucleotides \\
sets & \\
\hline
\end{tabular}

bretschneideri, Ricinus communis, Vitis vinifera, and Zea mays. To find salt stress-responsive TEs in common bean; leaf and root tissues were used. So, TEs differences between different tissues under the salt stress can be evaluated. 43 and 50 TEs were found in LC and LT samples respectively (Figure 2, Figure 3). Hit numbers of TONT2-I, EnSpm-2 EnSpm/CACTA, Copia-76, Copia-29, Copia-32, Copia-39, TONT2-LTR, MeloSINE-SINE, Copia21 and MuDR-118 TEs on genome were found to be the highest on LC sample (Figure 2) while hit numbers of Copia39_Copia, TONT2-I_Copia, Copia27_Copia, Copia29_Copia_- and Copia77_Copia TEs on genome were detected as the highest on LT sample (Figure 3). There was a significant difference of the TEs found in salt stress and control samples. A known feature of diverse retrotransposons is that they are turned on by stress conditions. Curiously, TEs can be differentially methylated under stress, dynamically organizing expression of neighboring genes, and not excepting defense response genes (Grandbastien, 1998; Dowen et al., 2012; Makarevitch et al., 2015). Similarly, induction of TEs in response to salt stress was shown for a noteworthy number of $P$. vulgaris genes. With this study, TEs related to salt stress, shown in Figure 2 and Figure 3, were detected first in leaf tissues of common bean.

Fifty and forty-six TEs were found in RC and RT samples, respectively (Figure 4, Figure 5). Hit numbers of MeloSINE-SINE, Copia-29 Copia, TONT2-I, piggyBac-4_piggyBac, Copia27_Copia, Copia29_Copia and Copia77_CopiaTEs on genome were found to be the most in $\overline{\mathrm{R}} \mathrm{C}$ samples (Figure 4) while hit numbers of TONT2-I_Copia, MeloSINESINE, Copia29_Copia, - Copia27_Copia, Copia32_Copia, Copia77_Copia and Copia11 TEs on genome were detected the most in RT samples (Figure 5). 


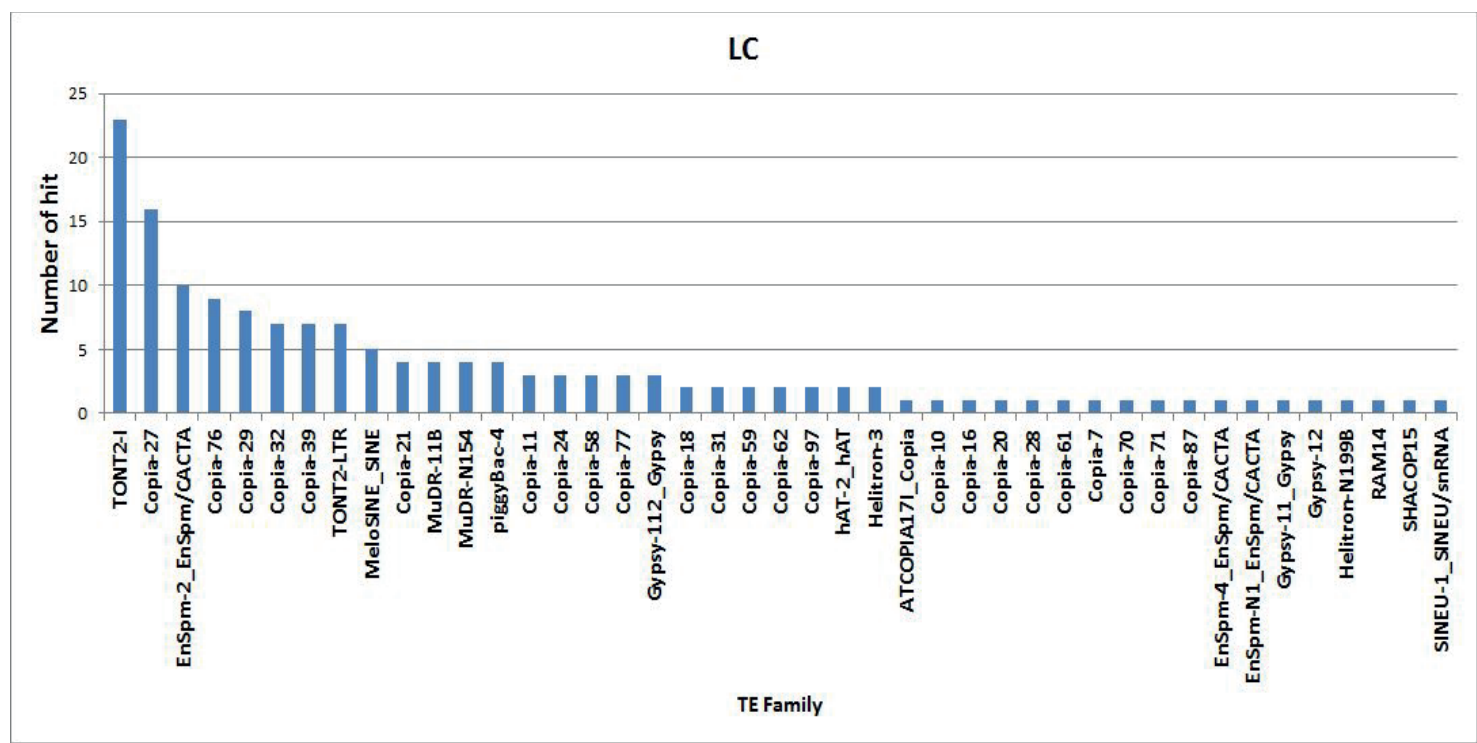

Figure 2. Transposable elements that hit on transcriptome reads of leaf control tissue (LC: leaf control, TE: transposable element)

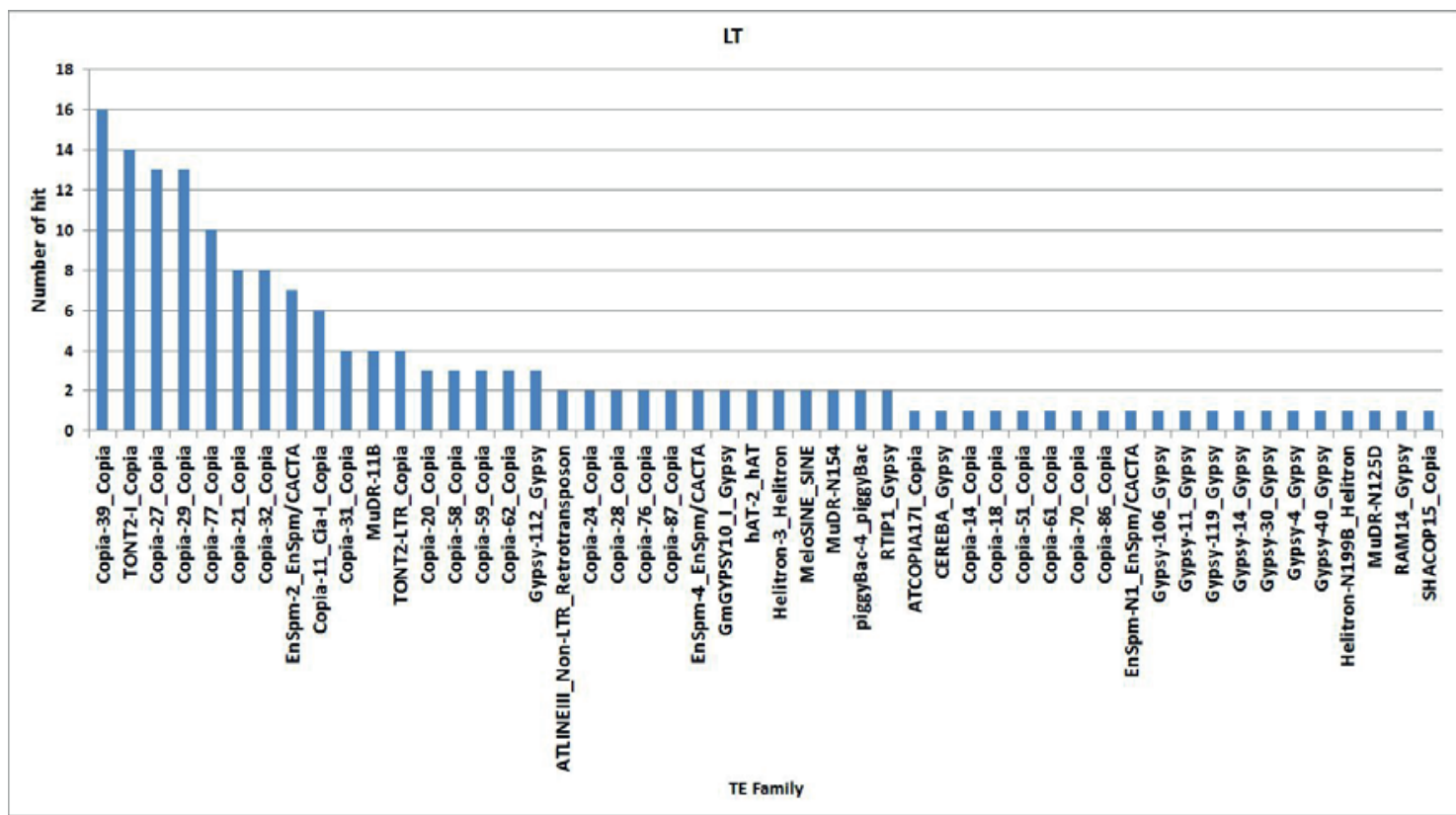

Figure 3. Transposable elements that hit on transcriptome reads of salt treated leaf tissue (LT: leaf treated, TE: transposable element)

There is an apparent difference in the most commonly found TEs in salt-treated and control samples of roots too. Additionally, roots and leaves exhibited quite different profiles on Venn diagram in terms of TEs response to salt stress (Figure 6). These results were supported by previous studies indicating TE dynamism in response to diverse environmental stresses (Makarevitch et al., 2015; Guo et al., 2015). Interestingly, TEs whose strength is inactivated by genome defense mechanisms can be re-activated when plants are subjected to abiotic stress conditions (Capy et al., 2000). It was noticed that TEs in salt-treated tissues were found to be more dynamic than in control samples. As reported in a previous study, activation of plant retrotransposons, such as Tnt 1 and Tto 1 by diverse stresses can be accepted consistently with above hypothesis (Weil and Wessler, 1990). Our analysis underlines the role of TEs in the evolutionary and environmental adaptation of common bean under salt stress. Moreover, TEs are kept silent by epigenetic mechanisms in optimum conditions (Feschotte et al., 2003). 
$\mathrm{RC}$

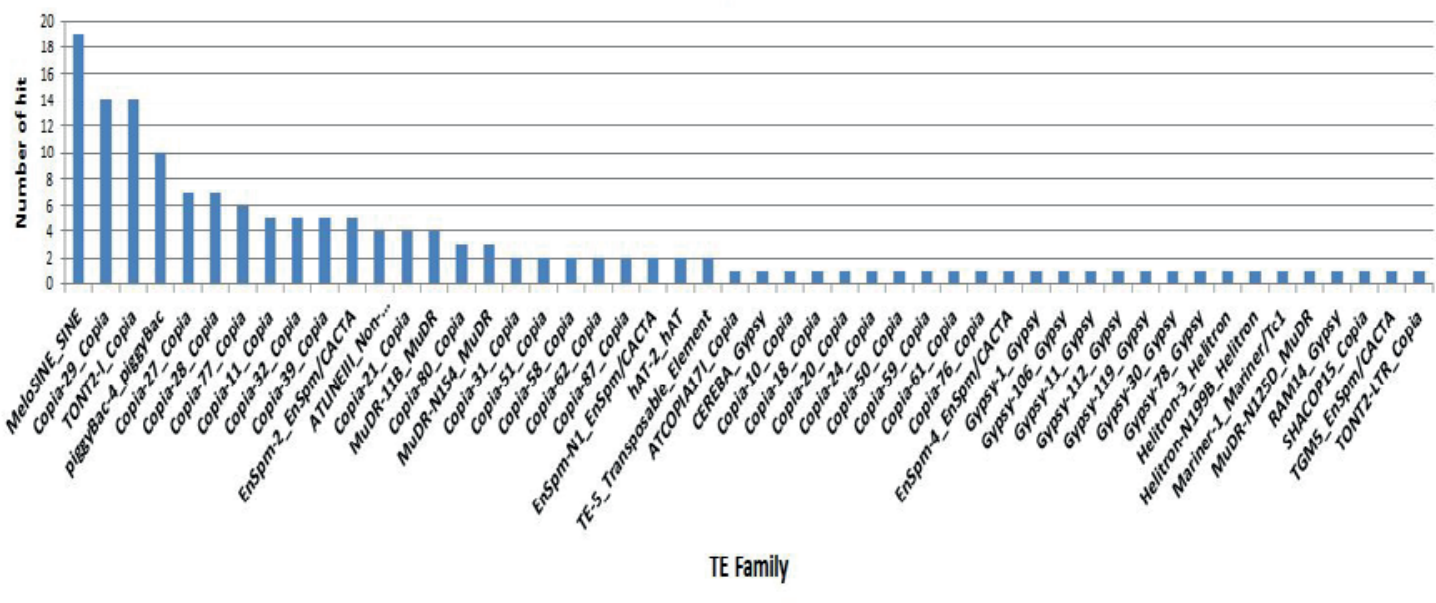

Figure 4. Transposable elements that hit on transcriptome reads of root control tissue (RC: root control, TE: transposable element)

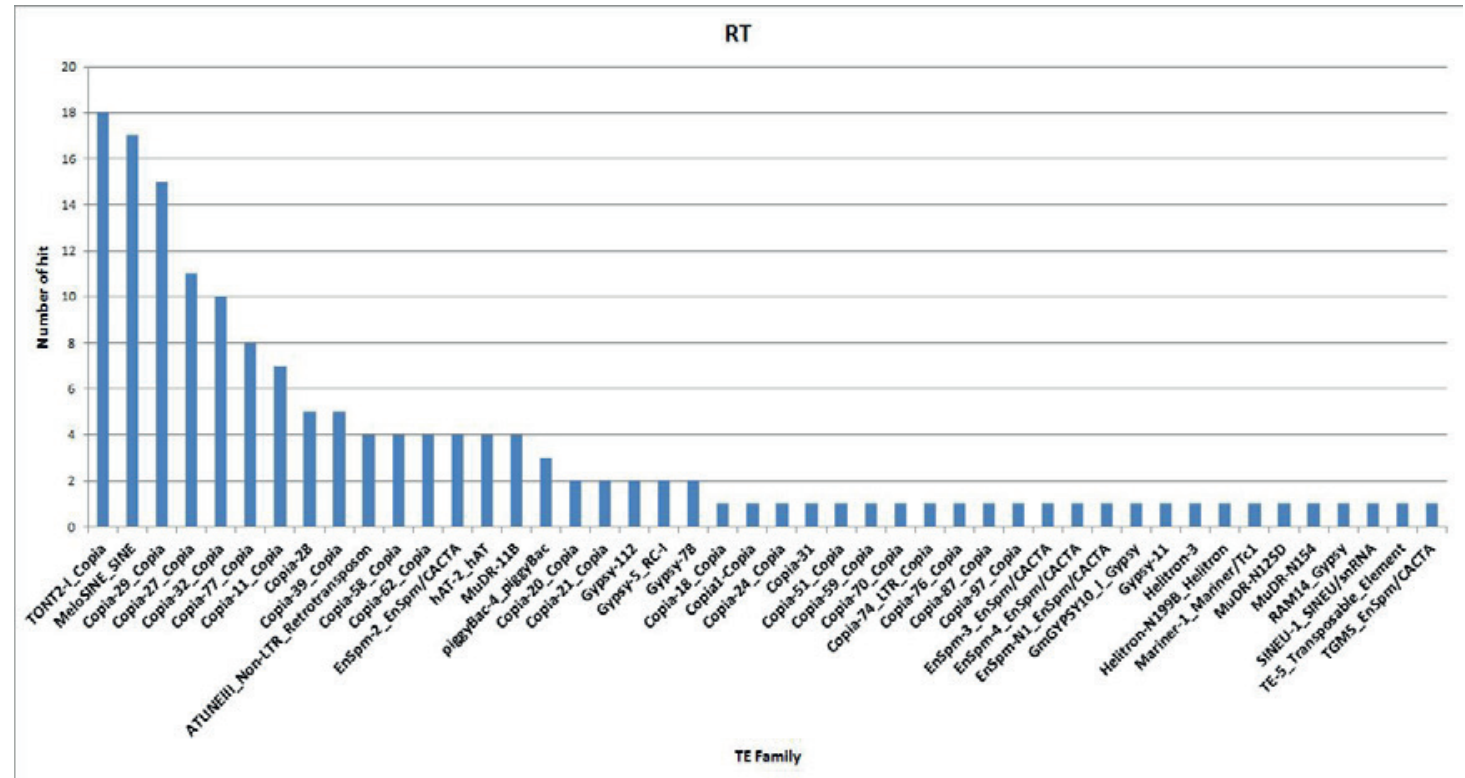

Figure 5. Transposable elements that hit on transcriptome reads of salt treated root (RT: root treated, TE: transposable element)

\subsection{GO analysis of TEs responsive to salt stress}

The cellular component classification after GO analysis of leaf tissues showed that the greatest numbers of gene products are located in cell part, cell, intracellular part, cytoplasm, intracellular of LC sample (Figure 7a). However, GO analysis of the most hit sequences of TE in LT sample were localized in the membrane, membrane part, cell part, cell, cytoplasm, integral components of the membrane, intrinsic components of the membrane, intracellular part and intracellular (Figure 7b). As a result of GO analysis of LC sample, 81 of 151 transcripts were found to be related to retrotransposons directly. In addition to this, alcohol dehydrogenase-like 7, auxin transport, Fbox WD-40 repeat and serine-threonine- kinase transcripts were also observed. On the other hand, arogenate dehydratase, prephenate dehydratase, chloroplastic-like bifunctional aspartate aminotransferase and glutamate aspartateprephenate aminotransferase, cell wall-associated hydrolase, Cysteine-rich RLK (receptor kinase) 8, integrase core domain containing, Regulator of rDNA transcription 15, RNA-directed DNA polymerase homolog, probable pectate lyase 18 , senescence-associated and UDP-galactose transporter 2-like transcripts that related to TEs were observed in salt-treated sample. In previous studies, it was reported that aspartate 


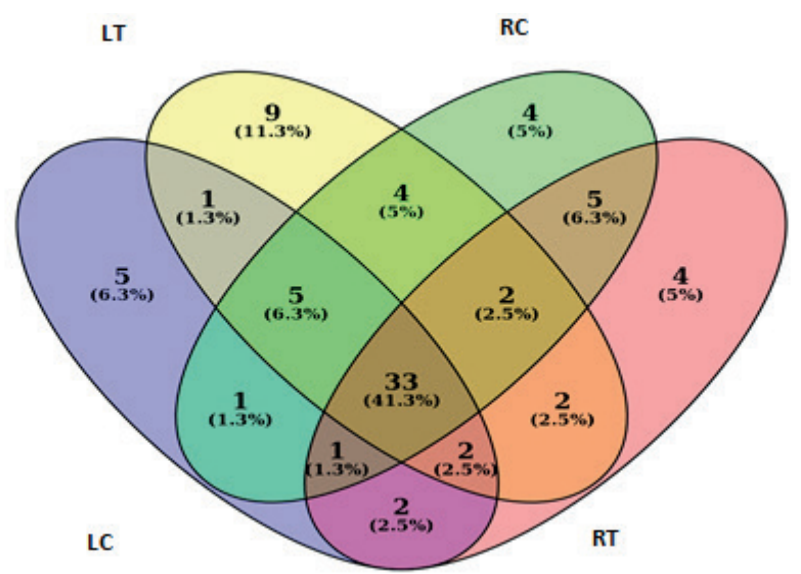

Figure 6. Venn diagrams of the distributions of transposable elements between leaf and root tissues LC: leaf control, LT: leaf treated, RC: root control, RT: root treated

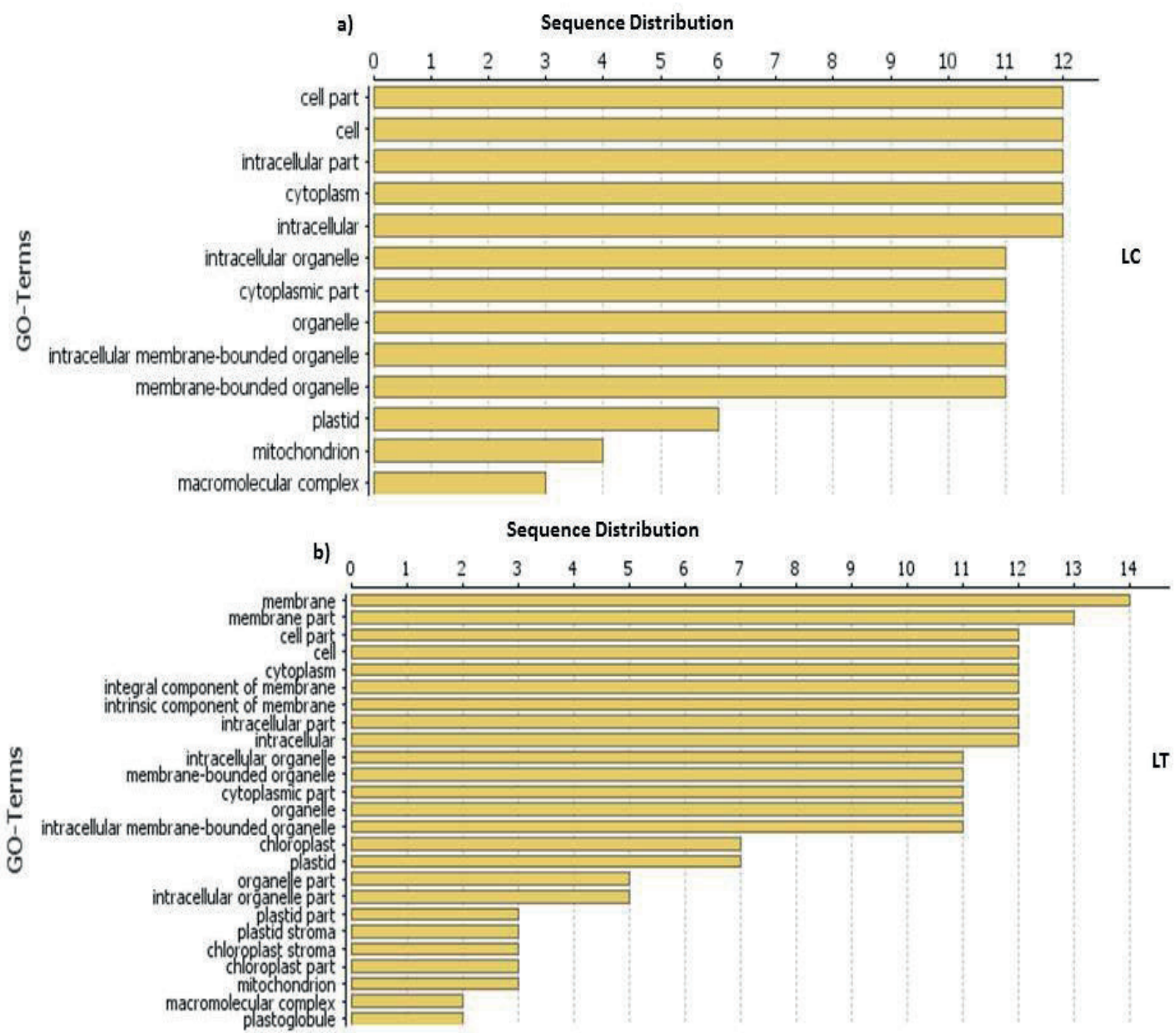

Figure 7. Gene ontology analysis of transposable elements found in leaf tissue (a: leaf control-LC, b: salt treated leaf-LT)

aminotransferase (AspAT) was induced during the addition of $\mathrm{NaCl}$ (Gao et al., 2013; Nam et al., 2012). These transcripts may have an important role in response to salt stress in P. vulgaris. Despite these genes, the regulatory functions of different TEs that have a role in salt response are not fully understood. This was the first characterization of different TEs responsive to salt stress.

The cellular component classification after GO analysis of root tissues showed the greatest number of gene products are located in cell part, membrane, cell, intracellular part, cytoplasm, intracellular of 
RC sample (Figure 8a). However, GO analysis of the most hit sequences of TE in RT sample were localized in cell part, cell, intracellular organelle, cytoplasm, organelle, and membrane-bounded organelle (Figure $8 \mathrm{~b}$ ). As a result of the $\mathrm{GO}$ analysis of RC samples, 69 of 145 transcripts were found related to retrotransposons directly. Additionally, alcohol dehydrogenase-like 7, arogenate dehydratase prephenate dehydratase chloroplasticlike, ATP synthase subunit beta, Bifunctional aspartate aminotransferase and glutamate aspartateprephenate aminotransferase, serine-threonine kinase and uncharacterized mitochondrial g00810like were found to be in large numbers. On the other hand, alcohol dehydrogenase-like 7, arogenate dehydratase prephenate dehydratase chloroplasticlike, Bifunctional aspartate aminotransferase and glutamate aspartate-prephenate aminotransferase, Reverse RNA-dependent DNA polymerase, serinethreonine kinase and tripartite motif-containing 65- like transcripts that related to TEs were observed in salt-treated root samples. When leaf and root tissues are considered in terms of TEs found and GO analysis, root tissue was found to be more induced under salt stress conditions.

As we find the importance of aspartate aminotransferase (AST), according to previous studies AST can catalyze the conversion of aspartate into glutamate, and it has quite elevated activity during salt stress (Ramanjulu et al., 1994). Brauc et al. (2011) reported that AST has an important role in amino acid metabolism, which can interact with plant defense response genes. The transcript of alcohol dehydrogenase is a key enzyme that induced under salt stress conditions in the plant (Zhang et al., 2016).

As a result of TEs and GO analysis, it was highlighted that certain families of transposons can play important roles in salt stress response in common bean.

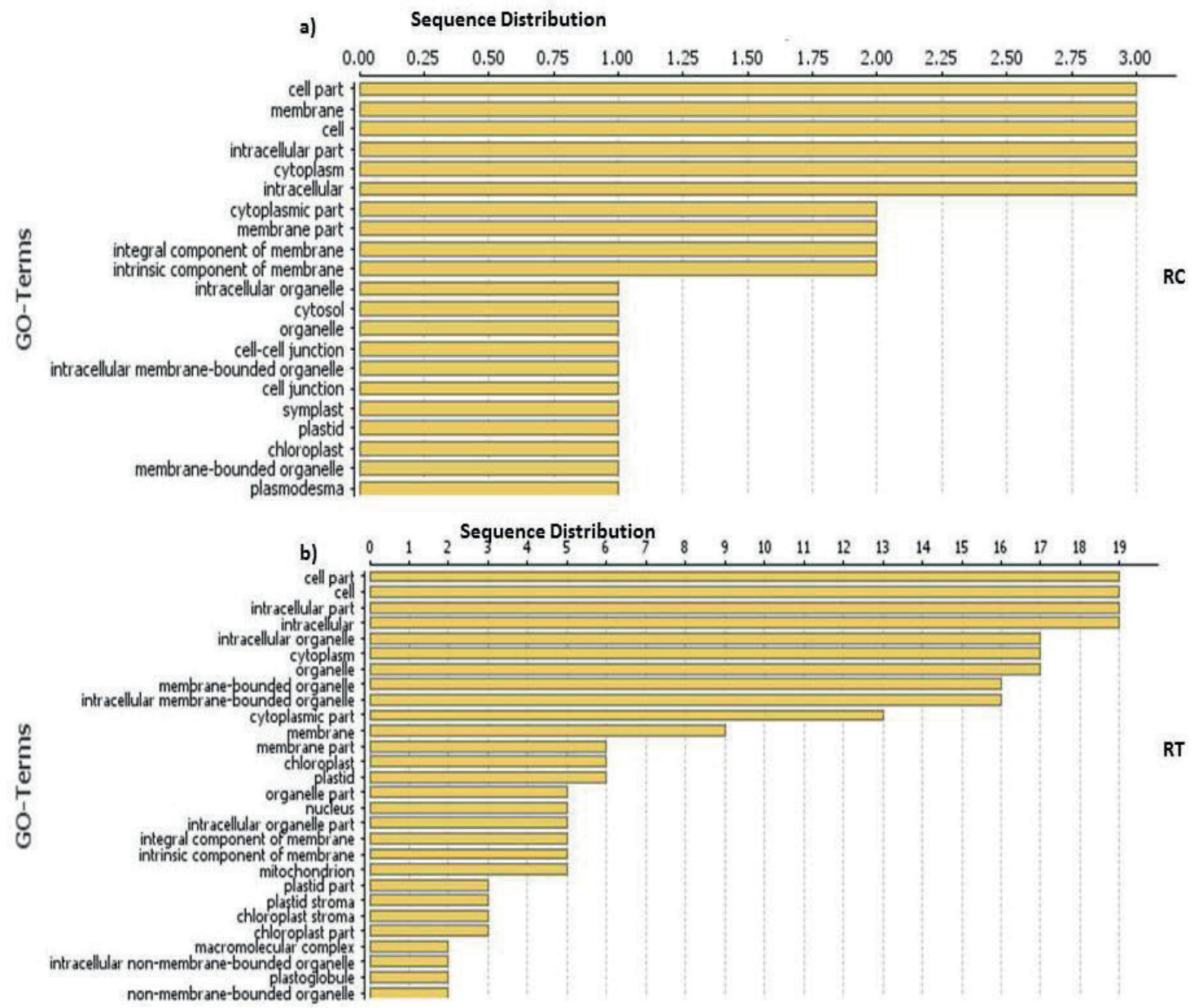

Figure 8. Gene ontology analysis of transposable elements found in root tissue (a: root control-RC, b: salt treated root-RT) 


\section{Conclusion}

The regulatory properties of the most wellcharacterized plant TEs show that these elements are quite important markers of stress responses of plants and could be used on different biotechnological applications. The fusion of LTR regions to reporter genes could represent a sensitive indicator of plant response to diverse stress conditions. Thus, future studies of RNA-Seq reads or next-generation sequencing applications will be needed to clearly detect the extent to which transcriptional activity of TEs regulate gene expression in response to various biotic and abiotic stress conditions.

\section{References}

Anonymous, 2017. VENNY, An Interactive Tool for Comparing Lists with Venn Diagrams BioinfoGP, CNB-CSIC by Juan C. Oliveros. (http://bioinfogp.cnb.csic.es/tools/venny/), (Date of access: 06.07.2017).

Beguiristain, T., Grandbastien, M.A., Puigdomènech, P., Casacuberta, J.M., 2001. Three Tnt1 subfamilies show different stress-associated patterns of expression in tobacco. Consequences for retrotransposon control and evolution in plants. Plant Physiology, 127(1): 212-221.

Biémont, C., 2010. A brief history of the status of transposable elements: from junk DNA to major players in evolution. Genetics, 186(4): 1085-1093.

Bitocchi, E., Nanni, L., Bellucci, E., Rossi, M., Giardini, A., Zeuli, P.S., Logozzo, G., Stougaard, J., McClean, P., Attene, G., 2012. Mesoamerican origin of the common bean (Phaseolus vulgaris L.) is revealed by sequence data. Proceedings of the National Academy of Sciences, 109(14): E788-E796.

Blair, M.W., Hurtado, N., Chavarro, C.M., MuñozTorres, M.C., Giraldo, M.C., Pedraza, F., Tomkins, J., Wing, R., 2011. Gene-based SSR markers for common bean (Phaseolus vulgaris L.) derived from root and leaf tissue ESTs: an integration of the BMc series. BMC Plant Biology, 11(1): 50-60.

Brauc, S., De Vooght, E., Claeys, M., Höfte, M., Angenon, G., 2011. Influence of over-expression of cytosolic aspartate aminotransferase on amino acid metabolism and defense responses against Botrytis cinerea infection in Arabidopsis thaliana. Journal of Plant Physiology, 168(15): 1813-1819.

Capy, P., Gasperi, G., Biémont, C., Bazin, C., 2000. Stress and transposable elements: co-evolution or useful parasites? Heredity, 85(2): 101-106.

Cavrak, V.V., Lettner, N., Jamge, S., Kosarewicz, A., Bayer, L.M., Scheid, O.M., 2014. How a retrotransposon exploits the plant's heat stress response for its activation. PLoS Genetics, 10(1): e1004115.

Conesa, A., Gotz, S., Garcia-Gomez, J.M., Terol, J., Talon, M., Robles, M., 2005. Blast2GO: a universal tool for annotation, visualization and analysis in functional genomics research. Bioinformatics, 21(18): 3674-3676.

Cowley, M., Oakey, R.J., 2013. Transposable elements re-wire and fine-tune the transcriptome. PLoS genetics, 9(1): e1003234.

De Souza, F.S., Franchini, L.F., Rubinstein, M., 2013. Exaptation of transposable elements into novel cisregulatory elements: is the evidence always strong? Molecular Biology and Evolution, 30(6): 1239-1251.

Dowen, R.H., Pelizzola, M., Schmitz, R.J., Lister, R., Dowen, J.M., Nery, J.R., Dixon, J.E., Ecker, J.R., 2012. Widespread dynamic DNA methylation in response to biotic stress. Proceedings of the National Academy of Sciences, 109(32): E2183-E2191.

Eichten, S.R., Ellis, N.A., Makarevitch, I., Yeh, C.-T., Gent, J.I., Guo, L., McGinnis, K.M., Zhang, X., Schnable, P.S., Vaughn, M.W., 2012. Spreading of heterochromatin is limited to specific families of maize retrotransposons. PLoS Genetics, 8(12): e1003127.

Feschotte, C., 2008. The contribution of transposable elements to the evolution of regulatory networks. Nature Reviews Genetics, 9(5): 397.

Feschotte, C., Swamy, L., Wessler, S.R., 2003. Genomewide analysis of mariner-like transposable elements in rice reveals complex relationships with stowaway miniature inverted repeat transposable elements (MITEs). Genetics, 163(2): 747-758.

Gao, S., Liu, K.-T., Chung, T.-W., Chen, F., 2013. The effects of $\mathrm{NaCl}$ stress on Jatropha cotyledon growth and nitrogen metabolism. Journal of Soil Science and Plant Nutrition, 13(1): 99-113.

Grandbastien, M.A., Lucas, H., More, J.B., Mhiri, C., Vernhettes, S., Casacuberta, J.M., 1997. The expression of the tobacco Tnt1 is linked to the plant defense responses. Genetica, 100(1):241-252.

Grandbastien, M.-A., 1998. Activation of plant retrotransposons under stress conditions. Trends in Plant Science, 3(5): 181-187.

Guo, Y., Singh, P.K., Levin, H.L., 2015. A long terminal repeat retrotransposon of Schizosaccharomyces japonicus integrates upstream of RNA pol III transcribed genes. Mobile DNA, 6(1): 19-26.

Haas, B.J., Papanicolaou, A., Yassour, M., Grabherr, M., Blood, P.D., Bowden, J., Couger, M.B., Eccles, D., Li, B., Lieber, M., 2013. De novo transcript sequence reconstruction from RNA-Seq: reference generation and analysis with Trinity. Nature Protocols, 8(8): 5069 .

Hiz, M.C., Canher, B., Niron, H., Turet, M., 2014. Transcriptome analysis of salt tolerant common bean (Phaseolus vulgaris L.) under saline conditions. PloS One, 9(3): e92598.

Hollister, J.D., Gaut, B.S., 2009. Epigenetic silencing of transposable elements: a trade-off between reduced transposition and deleterious effects on neighboring gene expression. Genome Research, 19(8): 14191428.

Ito, H., Gaubert, H., Bucher, E., Mirouze, M., Vaillant, I., Paszkowski, J., 2011. An siRNA pathway prevents 
transgenerational retrotransposition in plants subjected to stress. Nature, 472 (7341): 115-124

Ito, H., Yoshida, T., Tsukahara, S., Kawabe, A., 2013. Evolution of the ONSEN retrotransposon family activated upon heat stress in Brassicaceae. Gene, 518(2): 256-261.

Jurka, J., Kapitonov, V.V., Pavlicek, A., Klonowski, P., Kohany, O., Walichiewicz, J., 2005. Repbase Update, a database of eukaryotic repetitive elements. Cytogenetic and Genome Research, 110 (1):462-467.

Li, R., Hsieh, C.-L., Young, A., Zhang, Z., Ren, X., Zhao, Z., 2015. Illumina synthetic long read sequencing allows recovery of missing sequences even in the "finished" C. elegans genome. Scientific Reports, 5: $50-65$.

Li, X., Kong, Y., Zhao, Q.-Y., Li, Y.-Y., Hao, P., 2016. De novo assembly of transcriptome from nextgeneration sequencing data. Quantitative Biology, 4(2): 94-105.

Lin, J.-Y., Stupar, R.M., Hans, C., Hyten, D.L., Jackson, S.A., 2010. Structural and functional divergence of a $1-\mathrm{Mb}$ duplicated region in the soybean (Glycine max) genome and comparison to an orthologous region from Phaseolus vulgaris. The Plant Cell, 22(8): 2545 2561.

Makarevitch, I., Waters, A.J., West, P.T., Stitzer, M., Hirsch, C.N., Ross-Ibarra, J., Springer, N.M., 2015. Transposable elements contribute to activation of maize genes in response to abiotic stress. PLoS Genetics, 11(1): e1004915.

McClean, P.E., Mamidi, S., McConnell, M., Chikara, S., Lee, R., 2010. Synteny mapping between common bean and soybean reveals extensive blocks of shared loci. BMC Genomics, 11(1): 184-194.

McClintock, B., 1956. Controlling elements and the gene, Cold Spring Harbor symposia on quantitative biology. Cold Spring Harbor Laboratory Press, 21: 197-216.

McClintock, B., 1963. Further studies of gene-control systems in maize. Carnegie Institution Washington Year Book, 62: 486-493.
McClintock, B., 1984. The significance of responses of the genome to challenge. Science, 4676(226): 792801.

Nam, M.H., Huh, S.M., Kim, K.M., Park, W.W., Seo, J.B., Cho, K., Kim, D.Y., Kim, B.G., Yoon, I.I., 2012. Comparative proteomic analysis of early salt stressresponsive proteins in roots of SnRK2 transgenic rice. Proteome Science, 10(1): 25-44.

Pecinka, A., Dinh, H.Q., Baubec, T., Rosa, M., Lettner, N., Scheid, O.M., 2010. Epigenetic regulation of repetitive elements is attenuated by prolonged heat stress in Arabidopsis. The Plant Cell, 22(9): 3118 3129 .

Ramanjulu, S., Veeranjaneyulu, K., Sudhakar, C., 1994. Short-term shifts in nitrogen metabolism in mulberry Morus alba under salt shock. Phytochemistry, 37(4): 991-995.

Sigmaz, B., Agar, G., Arslan, E., Aydin, M., Taspinar, M.S., 2015. The role of the putrescine against the long terminal repeat (LTR) retrotransposon polymorphism induced by salinity stress in Triticum aestivum. Acta Physiologiae Plantarum, 37(11): 251-259.

Weil, C., Wessler, S., 1990. The effects of plant transposable element insertion on transcription initiation and RNA processing. Annual Review of Plant Biology, 41(1): 527-552.

Wicker, T., Sabot, F., Hua-Van, A., Bennetzen, J. L., Capy, P., Chalhoub, B., Flavell, A., Leroy, P., Morgante, M., Panaud, O., 2007. A unified classification system for eukaryotic transposable elements. Nature Reviews Genetics, 8(12): 973-982.

Yasuda, K., Ito, M., Sugita, T., Tsukiyama, T., Saito, H., Naito, K., Teraishi, M., Tanisaka, T., Okumoto, Y., 2013. Utilization of transposable element mPing as a novel genetic tool for modification of the stress response in rice. Molecular Breeding, 32(3): 505-516.

Zhang, F., Zhu, G., Du, L., Shang, X., Cheng, C., Yang, B., Hu, Y., Cai, C., Guo, W., 2016. Genetic regulation of salt stress tolerance revealed by RNA-Seq in cotton diploid wild species, Gossypium davidsonii. Scientific Reports, 6: 1-15. 